\title{
Development of complex of mathematical models at production stages of multilayer structures of spherical shape by polymerization method
}

\author{
Kozlov V.N. \\ Higher School of Cyber Physical Systems and Management \\ Peter the Great St. Petersburg Polytechnic University \\ (SPbPU) \\ St. Petersburg, Russia
}

\section{Akimov A.I.}

Department of economics, humanities and science

Orenburg branch of Gubkin Russian State University of Oil and Gas

Orenburg, Russia

akimovff11@mail.ru

\author{
Akimov I.A. \\ Department of mathematical analysis and mathematics \\ teaching technique \\ Orenburg State Pedagogical University (OSPU) \\ Orenburg, Russia \\ akimov_ia@mail.ru
}

Tugov V.V.

Department of Management and Informatics in Technical Systems

Orenburg State University (OSU)

Orenburg, Russia

sau@mail.osu.ru

\author{
Urban V.A. \\ Department of techno-spheric and information security \\ Orenburg State Agrarian University (OSAU) \\ Orenburg, Russia \\ urban.vladimir@mail.ru.
}

\begin{abstract}
In the article the developed mathematical models of heat exchange in the multilayer structures of spherical shape are presented. The mathematical description of the polymerization temperature process of composite materials is made at three production stages. Heat exchange is considered when the perfect coupling takes place between layers (boundary conditions of the fourth kind). Such products are widely used in the aviation industry and space technology. This work continues to research modeling of thermal physical processes in multilayer structures. The peculiarities of the application of the method of finite integral transformations and the method of isothermal surfaces for solving the set model problems are considered. Universality of the obtained mathematical apparatus allows creating software for carrying out experiments on computer facilities.
\end{abstract}

Keywords- heat exchange, polymerization, coupling conditions, multilayer structures

\section{INTRODUCTION}

The study of heat exchange processes is one of the main sections of modern research in the aviation, space and other industries [1,2]. As it is known, many production processes are to a greater or lesser extent related to changes in the temperature state and heat transfer. It should be noted that studies of the kinetics of a number of physical and chemical- technological processes are similar to the problems of stationary and non-stationary thermal conductivity.

Analytical theory of heat exchange is the scientific basic for the study of thermal processes proceeding in modern thermal-technical installations. Knowledge of the mechanisms of heat transfer allows to find the most optimal conditions for carrying out thermal processes, to create materials with set physical properties, to control technological processes of production, and to solve many other technical problems [3$12]$.

The problems of heat transfer for multilayer structures of spherical shape refer to problems with boundary conditions of the fourth kind. In connection with the development of hightemperature thermal physics, they become of primary importance when conducting thermal and strength calculations of the structures of aviation and space technology, calculations of multilayer composite materials widely used in many industries. It should be noted that analytical solutions are best suited for obtaining multilayer materials with set properties; they explicitly contain the basic physical parameters (thermal diffusivity, heat capacity, thermal conductivity, etc.).

In the article the mathematical models, that are informative in fundamental principles of heat exchange processes, are developed and investigated. To achieve the goal, it is proposed to apply the method of finite integral transformations and the method of isothermal surfaces that make it possible to solve 
problems connected with obtaining multilayer structures with regard to phase transitions.

\section{CHARACTERISTICS OF TECHNOLOGY}

In the process of multilayer structures production of spherical shape, it is necessary to observe certain technical and technological requirements, to take into account the heat release during polymerization. So, for example, if the heat emission at polymerization is not taken into account, this causes a change in the temperature mode of production and the appearance of residual thermal stresses and deformations during cooling.

The temperature polymerization process includes three stages (modes): I - preheating mode; II - polymerization; III cooling mode.

At the first stage, the temperature of the product rises from the temperature of the medium to the polymerization temperature under the effect of heat.

At the second stage, a polymerization front is formed, which moves inward the structure.

At the third stage, the finished product is cooled to the temperature of the medium.

At each stage, the speed rates of temperature increase and the corresponding temperature modes are determined. The peculiarity of thermal physical processes at each stage creates the need to use various mathematical models, in this case, at the beginning, models for the first and third stages are developed, and then models for the second stage are created on their basis.

\section{Statement and solution of the task at the first stage}

At the first stage of multilayer structures production by the polymerization method, non-interconnected heat and mass exchange takes place. Therefore, heat exchange and mass transfer are considered separately, although they are solved according to the same scheme. In this paper, let us consider heat exchange.

As it is known, the mathematical model of heat exchange in multilayer structures of a spherical shape is described by partial differential equations of parabolic type and have the form (central symmetry) (Fig. 1):

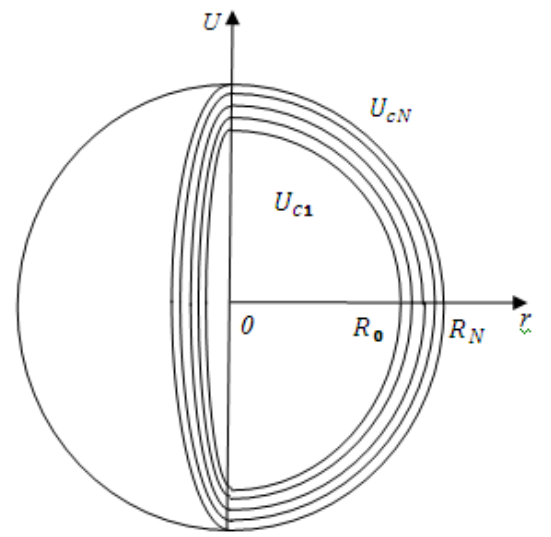

Fig. 1. N - layer sphere

$$
\begin{array}{r}
\frac{\partial U_{i}\left(r_{i}, \tau\right)}{\partial \tau}=a_{i}^{2}\left(\frac{\partial^{2} U_{i}\left(r_{i}, \tau\right)}{\partial r_{i}^{2}}+\frac{2}{r_{i}} \frac{\partial U_{i}\left(r_{i}, \tau\right)}{\partial r_{i}}\right)+\frac{q_{i}}{c_{i} \rho_{i}} \\
i=1,2, \ldots, N, R_{i-1} \leq r_{i} \leq R_{i}, \tau>0
\end{array}
$$

with initial conditions: $U_{i}\left(r_{i}, 0\right)=f_{i}\left(r_{i}\right)$; and boundary conditions:

$$
\begin{aligned}
& \lambda_{1} \frac{\partial U_{1}\left(R_{0}, \tau\right)}{\partial r_{1}}-\alpha_{1}\left[U_{1}\left(R_{0}, r\right)-U_{c 1}\right]=0 \\
& \lambda_{N} \frac{\partial U_{N}\left(R_{N}, \tau\right)}{\partial r_{N}}-\alpha_{N}\left[U_{N}\left(R_{N}, r\right)-U_{c N}\right]=0
\end{aligned}
$$

and with the coupling conditions:

$$
\begin{aligned}
U_{j}\left(R_{j}, \tau\right) & =U_{j+1}\left(R_{j}, \tau\right) \\
\lambda_{j} \frac{\partial U_{j}\left(R_{j}, \tau\right)}{\partial r_{j}} & =\lambda_{j+1} \frac{\partial U_{j+1}\left(R_{j}, \tau\right)}{\partial r_{j+1}} \\
j & =1,2, \ldots, N-1,
\end{aligned}
$$

where $U_{i}$ - temperature; $r_{i}$ - current radius of the layers; $\tau-$ time; $a_{i}^{2}$ - thermal diffusivity of the layer; $q_{i}$ - power of the source; $c_{i}$ - specific heat of the layer; $\rho_{\mathrm{i}}-$ material density of the layer; $N$ - number of layers; $R_{i}$ - radii of the layers; $\lambda_{i}-$ coefficient of thermal conductivity of layers; $R_{0}$ - radius of the surface of the inner layer; $\alpha_{i}$ - coefficient of thermal interaction of the surface layer with the medium; $U_{c 1}-$ temperature outside the inner layer; $R_{N}$ - radius of the surface of the outer layer; $U_{N}$ - temperature of the external surface of the product; $U_{c N}$ - temperature outside the outer layer of the product.

To solve the problems of thermal conductivity in multilayered bodies with inhomogeneous boundary conditions, we use the algebraic sum of the solution of the stationary problem of thermal conductivity with inhomogeneous boundary conditions and the non-stationary problem of thermal conductivity with homogeneous boundary conditions, because a direct solution of the original problem leads to slowly convergent series, which lengthens the calculations [13].

We seek the solution of the problem (1) - (5) in the form

$$
U_{i}\left(r_{i}, \tau\right)=S_{i}\left(r_{i}\right)+P_{i}\left(r_{i}, \tau\right), \quad i=1,2, \ldots, N \text {, }
$$

where $S_{i}\left(r_{i}\right)$ - solution of the stationary problem with inhomogeneous boundary conditions:

$$
\begin{gathered}
\frac{d^{2} S_{i}\left(r_{i}\right)}{d r_{i}^{2}}+\frac{2}{r_{i}} \frac{d S_{i}\left(r_{i}\right)}{d r_{i}}=0, \quad i=1,2, \ldots, N, \quad R_{i-1} \leq r_{i} \leq R_{i} ; \\
\lambda_{1} \frac{d S_{1}\left(R_{0}\right)}{d r_{1}}-\alpha_{1}\left[S_{1}\left(R_{0}\right)-U_{c 1}\right]=0 ; \\
\lambda_{N} \frac{d S_{N}\left(R_{N}\right)}{d r_{N}}-\alpha_{N}\left[S_{N}\left(R_{N}\right)-U_{c N}\right]=0 ; \\
S_{j}\left(R_{j}\right)=S_{j+1}\left(R_{j}\right) ; \lambda_{j} \frac{d S_{j}\left(R_{j}\right)}{d r_{j}}=\lambda_{j+1} \frac{d S_{j+1}\left(R_{j}\right)}{d r_{j+1}} ; \\
j=1,2, \ldots, N-1-\text { matching boundaries. }
\end{gathered}
$$

When solving the stationary problem (7) - (10), we use the method of finite integral transformations. 
$P_{i}\left(r_{i}, \tau\right)$-a solution of a non-stationary problem with homogeneous boundary conditions:

$$
\begin{gathered}
\frac{\partial P_{i}\left(r_{i}, \tau\right)}{\partial \tau}=a_{i}^{2}\left(\frac{\partial^{2} P_{i}\left(r_{i}, \tau\right)}{\partial r_{i}^{2}}+\frac{2}{r_{i}} \frac{\partial P_{i}\left(r_{i}, \tau\right)}{\partial r_{i}}\right)+\frac{q_{i}}{c_{i} \rho_{i}} \\
i=1,2, \ldots, N, R_{i-1} \leq r_{i} \leq R_{i}, \tau>0 ; \\
P_{i}\left(r_{i}, 0\right)=f_{i}\left(r_{i}\right)-S_{i}\left(r_{i}\right) ; \\
\lambda_{1} \frac{\partial P_{1}\left(R_{0}, \tau\right)}{\partial r_{1}}-\alpha_{1} P_{1}\left(R_{0}, \tau\right)=0 ; \\
\lambda_{N} \frac{\partial P_{N}\left(R_{N}, \tau\right)}{\partial r_{N}}-\alpha_{N} P_{N}\left(R_{N}, \tau\right)=0 ; \\
P_{j}\left(R_{j}, \tau\right)=P_{j+1}\left(R_{j}, \tau\right) ; \\
\lambda_{j} \frac{\partial P_{j}\left(R_{j}, \tau\right)}{\partial r_{j}}=\lambda_{j+1} \frac{\partial P_{j+1}\left(R_{j}, \tau\right)}{\partial r_{j+1}}
\end{gathered}
$$

$j=1,2, \ldots, N-1-$ matching boundaries.

The solution of problem (11) - (15) is obtained by the method of finite integral transformations.

As a result, the solution of the original problem (1) - (5) has the following form:

$$
U_{i}\left(r_{i}, \tau\right)=A_{i}+\frac{B_{i}}{r_{i}}+\sum_{n=1}^{\infty} \frac{c_{i n} \sin \left(\frac{\mu_{n} r_{i}}{a_{i}}+\varphi_{i, n}\right) e^{-\mu_{n}^{2} \tau} \sum_{m=1}^{N} \frac{\lambda_{m}}{a_{m}^{2}} c_{m, n}}{0,5 \sum_{m=1}^{N} \frac{\lambda_{m}}{a_{m}^{2}} c_{m, n}^{2}} \times
$$

$$
\times \frac{\int_{R_{m-1}}^{R_{m}}\left[f_{m}\left(r_{m}\right)-A_{m}-\frac{B_{m}}{r_{m}}\right] \sin \left(\frac{\mu_{n} r_{m}}{a_{m}}+\varphi_{m, n}\right) d r_{m}}{\left\{R_{m}-\frac{a_{m}}{\mu_{n}}\left[\sin \left(\frac{\mu_{n} R_{m}}{a_{m}}+\varphi_{m, n}\right) \cos \left(\frac{\mu_{n} R_{m}}{a_{m}}+\varphi_{m, n}\right)-\sin \left(\varphi_{m, n}\right) \cos \left(\varphi_{m, n}\right)\right]\right\}}
$$

where $\mu_{n}$ - roots of the characteristic equation;

$\varphi$ - angle of cross section of spherical shape product;

$B_{1}=\frac{U_{c N}-U_{c 1}}{\frac{\lambda_{1}}{R_{N}}\left(\frac{1}{\lambda_{n}}-\frac{1}{R_{N} \alpha_{N}}\right)-\frac{\lambda_{1}}{R_{0}}\left(\frac{1}{\lambda_{1}}+\frac{1}{R_{0} \alpha_{1}}\right)+\sum_{k=1}^{N-1} \frac{\lambda_{1}}{R_{k}}\left(\frac{1}{\lambda_{k}}-\frac{1}{\lambda_{k+1}}\right)} ;$

$$
\begin{gathered}
A_{1}=U_{c 1}-\frac{B_{1}}{R_{0}}\left(\frac{\lambda_{1}}{\alpha_{1} R_{0}}+1\right) ; \\
B_{i+1}=\frac{\lambda_{1}}{\lambda_{i+1}} B_{i} ; \\
A_{i+1}=A_{i}+\frac{B_{i}}{R_{i}}\left(1-\frac{\lambda_{i}}{\lambda_{i+1}}\right) .
\end{gathered}
$$

\section{STATEMENT AND SOLUTION OF THE TASK AT THE THIRD STAGE}

At the third stage of production of multilayer spherical structures, the multilayer sphere with central symmetry is cooled from the polymerization temperature to the ambient temperature (shop). Cooling starts from the outer surface of the product from the polymerization temperature of the binder to the ambient temperature (the electric heating is simply turned off and the product, together with the installation inside which it is, cools down to the shop temperature).

Under such assumptions, the formulation and solution of the heat transfer problem are the following: find the solution of the equation, taking into account the fact that the cooling progresses to the center of symmetry (Fig. 2):

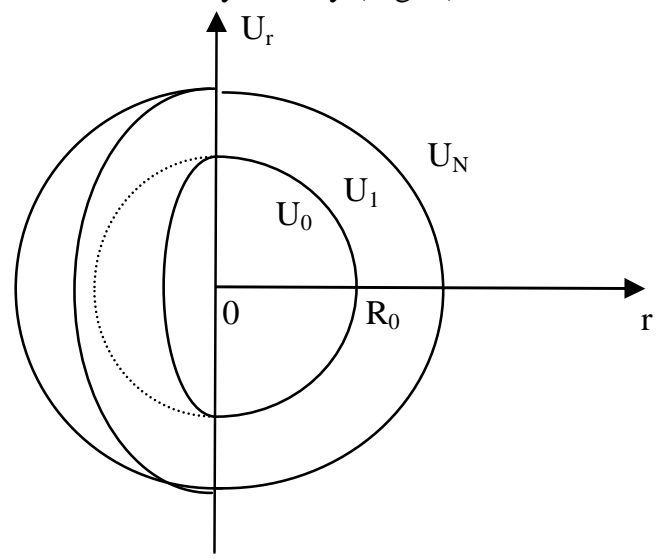

Fig. 2. Full sphere with polymerized layer

$$
\begin{gathered}
\frac{\partial U_{1}(r, \tau)}{\partial \tau}=a_{1}^{2}\left(\frac{\partial^{2} U_{1}(r, \tau)}{\partial r^{2}}+\frac{2}{r} \frac{\partial U_{1}(r, \tau)}{\partial r}\right), \tau>0, \\
R_{0} \leq r \leq R_{N} ; \\
\frac{\partial U_{2}(r, \tau)}{\partial \tau}=a_{2}^{2}\left(\frac{\partial^{2} U_{2}(r, \tau)}{\partial r^{2}}+\frac{2}{r} \frac{\partial U_{2}(r, \tau)}{\partial r}\right), \tau>0, \\
R_{N} \leq r \leq \infty ;
\end{gathered}
$$

with initial conditions: $U_{1}(r, 0)=f(r)=U_{1}$,

$$
U_{2}(r, 0)=U_{c}
$$

and boundary conditions:

$$
\begin{gathered}
-\lambda_{1} \frac{\partial U_{1}\left(R_{N}, \tau\right)}{\partial r}=-\lambda_{2} \frac{\partial U_{2}\left(R_{N}, \tau\right)}{\partial r} ; \\
U_{1}\left(R_{N}, \tau\right)=U_{2}\left(R_{N}, \tau\right),
\end{gathered}
$$

where $r$ - variable in the radial direction; $\lambda_{1}-$ thermal conductivity of the product; $\lambda_{2}$ - thermal conductivity of the medium; $U_{c}$ - temperature of the ambient medium (shop).

$\left(U_{c}<U(r, \tau)\right)$

We seek the solution of problem (21) - (25) in the form of stationary and non-stationary components:

$$
U(r, \tau)=S(r)+P(r, \tau)
$$

where $S_{i}\left(r_{i}\right)$ - solution of the stationary problem with inhomogeneous boundary conditions:

$$
\begin{gathered}
\frac{d^{2} S(r)}{d r^{2}}+\frac{2}{r_{i}} \frac{d S(r)}{d r}=0, R_{0} \leq r \leq R_{N} ; \\
S_{1}\left(R_{N}, \tau\right)=S_{2}\left(R_{N}, \tau\right) ;
\end{gathered}
$$




$$
-\lambda_{1} \frac{\partial S_{1}\left(R_{N}, \tau\right)}{\partial r}=-\lambda_{2} \frac{\partial S_{2}\left(R_{N}, \tau\right)}{\partial r} .
$$

The solution of the stationary problem is obtained using the method of finite integral transformations.

$P(r, \tau)$ - solution of the non-stationary problem with homogeneous boundary conditions:

$$
\begin{gathered}
\frac{\partial P(r, \tau)}{\partial \tau}=a_{1}^{2}\left(\frac{\partial^{2} P(r, \tau)}{\partial r^{2}}+\frac{2}{r_{i}} \frac{\partial P(r, \tau)}{\partial r}\right), \tau>0, \\
R_{0} \leq r \leq R_{N}
\end{gathered}
$$

with initial conditions: $P(r, 0)=f(r)-S(r)$;

and boundary conditions: $\lambda_{1} \frac{\partial P\left(R_{0}, \tau\right)}{\partial r}-\alpha_{1} P\left(R_{0}, \tau\right)=0$;

$$
\lambda_{1} \frac{\partial P\left(R_{N}, \tau\right)}{\partial r}-\alpha_{N} P\left(R_{N}, \tau\right)=0 .
$$

The solution is obtained by the method of finite integral transformations.

Passing to the original, we obtain the solution of the original problem (21) - (25) in the form:

$$
U(r, \tau)=A+\frac{B}{r}+\sum_{n=1}^{\infty} \frac{c_{1 n} \sin \left(\frac{\mu_{n} r}{a_{1}}+\varphi_{1, n}\right) e^{-\mu_{n}^{2} \tau} \frac{\lambda_{1}}{a_{1}^{2} c_{1, n}}}{0,5 \frac{\lambda_{1}}{a_{1}^{2}} c_{1, n}^{2}} \times
$$

$$
\times \frac{\int_{R_{0}}^{R_{N}} r\left[f(r)-A-\frac{B}{r}\right] \sin \left(\frac{\mu_{n} r}{a_{1}}+\varphi_{1, n}\right) d r}{\left\{r-\frac{a_{1}}{\mu_{n}}\left[\sin \left(\frac{\mu_{n} r}{a_{1}}+\varphi_{1, n}\right) \cos \left(\frac{\mu_{n} r}{a_{1}}+\varphi_{1, n}\right)-\sin \left(\varphi_{1, n}\right) \cos \left(\varphi_{1, n}\right)\right]\right\}},
$$

where $B=\frac{U_{c N}-U_{1 N}}{\frac{\lambda_{1}}{R_{N}}\left(\frac{1}{\lambda_{1}}-\frac{1}{R_{N} \alpha_{1}}\right)-\frac{\lambda_{1}}{R_{0}}\left(\frac{1}{\lambda_{1}}+\frac{1}{R_{0} \alpha_{1}}\right)}$;

$$
A=U_{1}-\frac{B_{1}}{R_{0}}\left(\frac{\lambda_{1}}{\alpha_{1} R_{0}}+1\right) \text {. }
$$

\section{STATEMENT AND SOLUTION OF THE TASK AT THE SECOND STAGE}

To solve the problem of the second stage, we use the method of isothermal surfaces, as well as mathematical models of the problems of the first (1) and the third stages and their analytical solutions.

The mathematical model of heat exchange in a polymerized layer (the central symmetry) of a product of a hollow spherical shape has the form:

$$
\begin{gathered}
\frac{\partial U_{1}(r, \tau)}{\partial \tau}=a_{1}^{2}\left(\frac{\partial^{2} U_{1}(r, \tau)}{\partial r^{2}}+\frac{2}{r} \frac{\partial U_{1}(r, \tau)}{\partial r}\right), \quad \tau>0 \\
R_{0} \leq r \leq R_{N}
\end{gathered}
$$

$$
\begin{gathered}
\frac{\partial U_{2}(r, \tau)}{\partial \tau}=a_{2}^{2}\left(\frac{\partial^{2} U_{2}(r, \tau)}{\partial r^{2}}+\frac{2}{r} \frac{\partial U_{2}(r, \tau)}{\partial r}\right), \tau>0, \\
R_{N} \leq r \leq \infty
\end{gathered}
$$

with initial conditions: $U_{1}(r, 0)=f(r)=U_{1}$,

$$
U_{2}(r, 0)=U_{c} \text {; }
$$

and boundary conditions:

$$
\begin{gathered}
-\lambda_{1} \frac{\partial U_{1}\left(R_{N}, \tau\right)}{\partial r}=-\lambda_{2} \frac{\partial U_{2}\left(R_{N}, \tau\right)}{\partial r} ; \\
U_{1}\left(R_{N}, \tau\right)=U_{2}\left(R_{N}, \tau\right) .
\end{gathered}
$$

The analytical solution of the problem of the third stage of production is sought in the form of stationary and nonstationary components:

$$
\begin{aligned}
& U(r, \tau)=S(r)+P(r, \tau) . \\
& S(r, \tau)=U_{1}(r, \tau)-\frac{B}{R_{0}}\left(\frac{\lambda_{1}}{\alpha_{1} R_{0}}+1\right),
\end{aligned}
$$

where

$$
\begin{gathered}
B=\frac{U_{c N}-U_{1 N}}{\frac{\lambda_{1}}{R_{N}}\left(\frac{1}{\lambda_{1}}-\frac{1}{R_{N} \alpha_{1}}\right)-\frac{\lambda_{1}}{R_{0}}\left(\frac{1}{\lambda_{1}}+\frac{1}{R_{0} \alpha_{1}}\right)} . \\
P(r, \tau)=\sum_{n=1}^{\infty} \frac{c_{1, n} \sin \left(\frac{\mu_{n} r}{a_{1}}+\varphi_{1, n}\right) e^{-\mu_{n}^{2} \tau} \frac{\lambda_{1}}{a_{1}^{2}} c_{1, n}}{0,5 \frac{\lambda_{1}}{a_{1}^{2}} c_{1, n}^{2}} \times
\end{gathered}
$$

$$
\times \frac{\int_{R_{0}}^{R_{N}} r\left[f(r)-A-\frac{B}{r}\right] \sin \left(\frac{\mu_{n} r}{a_{1}}+\varphi_{1, n}\right) d r}{\left\{r-\frac{a_{1}}{\mu_{n}}\left[\sin \left(\frac{\mu_{n} r}{a_{1}}+\varphi_{1, n}\right) \cos \left(\frac{\mu_{n} r}{a_{1}}+\varphi_{1, n}\right)-\sin \left(\varphi_{1, n}\right) \cos \left(\varphi_{1, n}\right)\right]\right\}} .
$$

For the mathematical modeling and solution of the nonstationary problem of heat exchange, the isothermal surface method is used at the second stage of production of multilayer constructions of a hollow spherical shape [14].

The main point of this method consists in replacing the true temperature distribution $U_{k, n}$ inside each area $D_{k, \tau}$ by not stationary, at fixed positions of the boundary $S_{i, \tau}: \xi_{i, n}=\xi_{i}\left(\tau_{n}\right), n=1,2,3 \ldots$

Fixing arbitrarily the positions of the boundary $S_{i, \tau}: \xi_{i, n}=\xi_{i}\left(\tau_{n}\right)$, we replace the area of continuous change of this boundary by a discrete set $\left\{\xi_{i, n}\right\}$, representing the increasing and bounded above sequence.

In areas $D_{i}, \tau_{n}=\left\{0<r<\xi_{i}, n\right\}$, we find the non-stationary temperature distribution $U_{k, n}(r, \tau)$ and using them, the function of a continuous argument $\tau=\tau\left(\xi_{i}\right)$, is replaced by a function of a discrete argument $\tau_{i}=\tau\left(\xi_{i, n}\right)$. 
Due to this, a discrete spectrum is allocated in the continuous spectrum of the Eigen values of the problem. This allows selecting and normalizing the minimal system of Eigen functions in the continuous spectrum in the corresponding domains. The obtained values $U_{k, n}(r, \tau)$ of the approximate one satisfy the condition of the problem for $\tau=\tau_{j}$ (see. Fig.3).

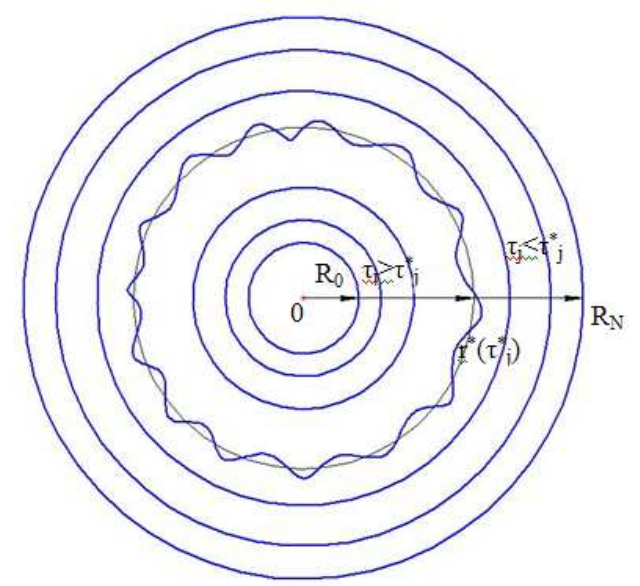

Fig. 3. The movement of the boundary of the polymerization zone in the spherical layer $\left|r^{*}\left(\tau_{j}^{*}\right)=\xi\left(\tau_{j}^{*}\right)=R_{N}-V r_{j}^{*}\right| \tau=\tau_{j}$.

Speed $V$ of polymerization boundary movement is experimentally determined for each type of binder. This makes it possible to determine approximately the positions of the polymerization boundary for each instant of time $\tau_{j}$ according to the formula:

$$
r\left(\tau_{j}^{*}\right)=\xi\left(\tau_{j}^{*}\right)=R_{N}-V r_{j}^{*}
$$

where $\tau_{j}^{*}$ - critical time corresponding to the position of the polymerization boundary.

The temperature in the autoclave at the second stage of composites production corresponds to the polymerization temperature of the selected type of binder, that is, until the completion of the second stage of production remains constant.

The main point of the method of isothermal surfaces is that the continuous spectrum of the polymerization boundary moving $R_{0} \leq r\left(\tau_{j}\right) \leq R_{N}$, is replaced by a discrete $\operatorname{set}\left\{r^{*}\left(\tau_{j}^{*}\right)\right\}=\left\{\xi\left(\tau_{j}^{*}\right)\right\}$ (the position of the polymerization boundary at fixed moments of time $\tau_{j}^{*}$ ).

These fixed uneven lines (Fig. 3) are marked by concentric circles.

Thus, the method of isothermal surfaces makes it possible to trace the nonlinear problem of non-stationary thermal conductivity to two already solved linear problems in intervals $r_{j}^{*} \leq r \leq R_{N}$ and $R_{0} \leq r \leq r_{j}^{*}$ using the method of finite integral transformations.

Consider three positions of arbitrary time $\tau_{j}$ and critical time $\tau_{j}^{*}$ :

$$
\text { 1) } \tau_{j}<\tau_{j}^{*} \text {;2) } \tau_{j}=\tau_{j}^{*} \text {; 3) } \tau_{j}>\tau_{j}^{*} \text {. }
$$

When $\tau_{j}<\tau_{j}^{*}$, the area under consideration (see Fig. 3) coincides with the area of complete polymerization; consequently, the mathematical model of the second stage of the process coincides with (35) - (39) and the solution of the problem has the form (40) - (43).

When $\tau_{j}=\tau_{j}^{*}$, a temperature value $U\left(r^{*}, \tau_{j}^{*}\right)$ coincides with the temperature of intensive polymerization of the binder, that is, with the temperature of the second stage of spherical composites production.

When $\tau_{j}>\tau_{j}^{*}$, the considered area coincides with the nonpolymerized multilayered area (see Fig. 3). Consequently, the mathematical model coincides with (1) - (5) and the problem in this zone is solved by the method of integral transformations and has the form (16).

\section{CONCLUSION}

Thus, the obtained complex of mathematical models allows one, with a certain degree of accuracy, to investigate the dynamics of heat exchange in multilayer structures of spherical shape under the influence of given and time-varying temperatures and boundary conditions. With the help of analytical solutions, it is possible to estimate the temperature distribution in each layer of the product. The application of the method of finite integral transformations and the method of isothermal surfaces made it possible to simulate the spatiotemporal dependences of thermal fields in multilayer structures with specific thermal physical properties in analytical and numerical form, using finite-difference schemes.

\section{References}

[1] V.N. Kozlov, I.A. Akimov, Management of power systems. Part 5. Mathematical models and differential problems of thermal physic processes in multilayer constructions with phase transitions, St. Petersburg, 2007, p. 204.

[2] V.V. Tugov, I.A. Akimov, "Study of technologies errors influence on the operational characteristics of avia constructions made from composite materials", The Volga Scientific and Technical Bulletin, vol. 1, pp. 58-61, january 2016.

[3] M. El Youbi, M. Rougui, "Modeling the Effect of Boundary Conditions on the Stability of Multilayer Composite Structures: Case of Buckling Bifurcation. Analytical and Numerical Results Using "SHELL 181" Element", International Review of Mechanical Engineering (IREME), vol. 9 (3), pp. 307-313, 2015.

[4] P. Ravindra, P. Palani, "Active Vibration Control in Smart Structures", International Review of Mechanical Engineering (IREME), vol. 8 (2), pp. 379-386, 2014

[5] A.A. Safonov, B.A. Shcheglov, "Mathematical modeling of polymerization process at production of polymeric composite materials", Problems of mechanical engineering and reliability of cars, vol. 6, pp. 55-60, 2011.

[6] V.A. Kudinov, E.M. Kartashov, V.V. Kalashnikov, Analytical solutions of problems of heatmass transfer and thermoelasticity for multilayered designs: manual for higher education institutions, Moscow: Higher School, 2005, p. 430.

[7] Y. Yener and M. N. Ozisik, "On the solution of unsteady heat conduction in multi-region finite media with time dependent heat transfer 
coefficient", in Proceedings of the 5th International Heat Transfer Conference, Tokyo, pp. 188-192, 1974.

[8] F.B. Yeh, "Prediction of the Transient and Steady Temperature Distributions in a Two-Layer Composite Slab in Contact With a Plasma: Exact Closed-Form Solutions", J. Phys. D: Appl. Phys., 40, pp. 36333643.

[9] S. Singh, P.K. Jain, and Rizwan-uddin "Analytical Solution to Transient Heat Conduction in Polar Coordinates With Multiple Layers in Radial Direction", Int. J. Therm. Sci., 47, pp. 261-273. Int. J. Therm. Sci. 47 (2008) 261-273.

[10]N.D. Milosevic, M. Raynaud, "Analytical solution of transient heat conduction in a two-layer anisotropic cylindrical slab excited superficially by a short laser pulse", International Journal of Heat and Mass Transfer, vol. 47, pp. 1627-1641, 2004.

[11] X. Lu, P. Tervola, M. Viljanen, "Transient Analytical Solution to Heat Conduction in Composite Circular Cylinder", Int. J. Heat Mass Transfer, vol. 49, pp. 341-348, 2006.

[12] A.V. Lykov, Yu.A Mikhaylov, Theory of heat and mass transfer. Moscow, 1963. p. 534.

[13]E.N. Tugolukov, Solution of thermal conductivity problems by the method of finite integral transformations. Tambov: Publishing House of the STU, p. 174, 2005.

[14]M.N. Shafeev, "On the convergence of the method of isothermal surfaces", VINITI, vol. 6597-1386. Ufa, p. 12, 1986. 